\title{
La Configuración de una Comunidad de Investigadores Educativos en una Universidad Pública Mexicana: de la Individualidad a la Colaboración
}

\author{
Patricia Moreno(1), Margarita Campos ${ }^{(2)}$ y Guillermo Rodríguez ${ }^{(1)}$ \\ (1) Facultad de Derecho y Ciencias Sociales, Ciudad Universitaria, Benemérita Universidad Autónoma de \\ Puebla. Av. San Claudio y 22 Sur, Col. San Manuel, Puebla, Puebla, México. \\ (e-mail: pmr1993@hotmail.com; guillorodriguez81@hotmail.com) \\ (2) Facultad de Medicina, Benemérita Universidad Autónoma de Puebla, Av. 13 Sur 2702, Col. Volcanes, \\ Puebla, Puebla, México. (e-mail: armycampos@gmail.com).
}

Recibido Oct. 26, 2015; Aceptado Dic. 15, 2015; Versión final Mar. 10, 2016, Publicado Oct. 2016

\begin{abstract}
Resumen
El objetivo del estudio es explorar la configuración de una comunidad de investigadores educativos en una universidad pública mexicana, la Benemérita Universidad Autónoma de Puebla (BUAP). Los referentes para determinar cómo se ha formado dicha comunidad son el Consejo Mexicano de Investigación Educativa (COMIE), el Sistema Nacional de Investigadores (SNI) y el Programa para el Desarrollo Profesional Docente de Tipo Superior (PRODEP- Cuerpos Académicos). El COMIE es una asociación civil cuyas actividades han dado cuenta de los principales problemas y propuestas de solución en el campo educativo en todos los niveles. En el caso del SNI y de PRODEP son instancias gubernamentales que apoyan y reconocen el trabajo individual y grupal de los académicos. Los registros de las tres fuentes de información (COMIE, PRODEP y SNI) permiten afirmar que la comunidad de investigadores educativos en la BUAP ha tenido un crecimiento significativo.
\end{abstract}

Palabras clave: investigación educativa; cuerpos académicos; líneas de investigación, investigación científica

\section{The Configuration of a Community of Education Researchers in a Mexican Public University: from Individuality to Collaboration}

\begin{abstract}
The objective of this study is to explore the configuration of a community of education researchers in a public Mexican university, the Benemérita Universidad Autónoma de Puebla (BUAP). The references to determine how this community was formed are the Mexican Council of Educational Research (COMIE), the National System of Researchers (SNI) and the Professional Higher Education Development Program (PRODEP - Academic Groups). The COMIE is a civil association that with its activities has revealed the main problems and solutions at all levels of the educational field. In the cases of SNI and PRODEP, they are government bodies that support and recognize the individual and group work of academic personnel. The records of the three sources of information (COMIE, PRODEP and SNI) allow declaring that this community of education researchers of the BUAP has had a meaningful growth.
\end{abstract}

Keywords: education research; education researcher; academic groups; lines of research; scientific research 
La Configuración de una Comunidad de Investigadores Educativos en una Universidad Pública Moreno

\section{INTRODUCCIÓN}

Si se atiende a un principio histórico la investigación educativa en México tiene sus orígenes por el impulso de la Organización para la Educación, la Ciencia y la Cultura de Naciones Unidas (UNESCO) que en la década de los años cincuenta, apoyó la creación de dos instituciones el Centro Regional para la Educación Funcional y Alfabetización para América Latina (CREFAL) fundado en 1951 y el Instituto Latinoamericano de Comunicación Educativa (ILCE) creado en 1956. Sin embargo, se argumenta que la investigación en estos centros fue una actividad secundaria.

En la década de los años sesenta se fundaría uno de los espacios de investigación, hasta la fecha, más respetados en el ámbito educativo, el Centro de Estudios Educativos (CEE) que inició sus actividades en 1964. Según datos del Consejo Mexicano de Investigación Educativa (COMIE) el CEE fue el primer centro moderno de investigación multidisciplinaria. Sus investigaciones apuntaron centralmente a diagnósticos del sistema educativo nacional e inauguraron la primera revisión crítica de las estadísticas educativas nacionales desde la perspectiva de la equidad. Este Centro promovió también proyectos de investigaciónacción comprometidos con los pobres (COMIE, 2003).

Para Martínez (1996) la década de los años setenta es la "primera etapa de consolidación" debido al número de centros de investigación que se crearon al interior de instituciones como la Universidad Nacional Autónoma de México (UNAM) y del Instituto Politécnico Nacional (IPN). Atendiendo a los estados del conocimiento del COMIE, en la UNAM se fundaron la Comisión de Nuevos Métodos de Enseñanza y el Centro de Didáctica, integrándose después al Centro de Estudios sobre la Universidad, actualmente Instituto de Investigaciones sobre la Universidad y la Educación (IISUE). Este último es el centro de investigación educativa más grande del país.

En el Centro de Investigación y Estudios Avanzados (CINVESTAV) inicialmente participaron en la reforma del plan de estudios de la primaria y en la elaboración de los libros de textos gratuitos para dicho nivel, en ciencias naturales y en matemática respectivamente. En 1976 iniciaron sus programas de investigación y de maestría y en los noventa los doctorados para los externos (COMIE, 2003). En los años ochenta, otras universidades estatales y del sector privado realizan investigación educativa, entre las primeras, se puede mencionar a la universidad de Guadalajara, a la Universidad Veracruzana, la Universidad de Aguascalientes, la Universidad de Yucatán, la Universidad de Puebla, así como las universidades de Baja California y Morelos. Entre las universidades particulares destaca la Universidad Iberoamericana, institución que dirige la Compañía de Jesús con sedes en Ciudad de México, León, Jalisco, Puebla y Tijuana (COMIE, 2003). Las escuelas normales espacios educativos donde se forman a los profesores de educación básica reportaban escasa investigación educativa.

Otras instancias han realizado investigación educativa, aunque no sistemáticamente como la Secretaría de Educación Pública. Siguiendo tal idea se pueden considerar en este apartado el Centro Nacional de Evaluación (CENEVAL), asociación civil creada en 1994 quien ha sido responsable de diseñar y aplicar pruebas estandarizadas cuyo propósito es valorar los conocimientos, habilidades y competencias en el ámbito de la educación media y también superior, así como ofrecer seminarios para poder certificar competencias en el ámbito laboral. Los resultados que obtiene son reportados a las instituciones educativas que contratan sus servicios. La institución lleva a cabo actividades de investigación como el Proyecto 12x2 que busca validar la prueba EXANI-1 en los niveles de educación media superior y superior (CENEVAL, 2016). En este esfuerzo de síntesis, se incluye al Instituto Nacional de Evaluación Educativa (INEE) creado en el año 2002 por decreto presidencial. En el año 2013 se transformó en un organismo público autónomo con personalidad jurídica y patrimonio propio. Entre sus principales tareas se encuentra evaluar al Sistema Educativo Nacional en los niveles de preescolar, primaria, secundaria y educación media superior (INNE, 2015). El Instituto actualmente mantiene relación con algunas redes de investigadores que le permiten llevar a cabo trabajo colaborativo.

Según Altbach (2007) la universidad sigue manteniéndose como uno de los lugares más efectivos para realizar investigación de todo tipo, de manera especial, las llamadas universidades de investigación. Estas últimas forman parte de los sistemas educativos de naciones altamente desarrolladas. Albatch (2007) afirma que es común en países industrializados que los fondos de apoyo a la investigación procedan tanto de los gobiernos como de donaciones de fundaciones y de empresas. En el caso de los países con menos posibilidades de desarrollo económico el financiamiento proviene principalmente de los gobiernos por medio de las políticas que para ello se diseñan. Debido a ello la investigación queda suscrita a una lógica de control y administración estatal. En ese sentido, México no es la excepción. Siguiendo tal idea, el interés de esta investigación es explorar cómo se ha configurado la comunidad de investigadores educativos de la Benemérita Universidad Autónoma de Puebla en México, sobre todo, tomando en cuenta la incidencia que han tenido el Consejo Nacional de Ciencia y Tecnología (Sistema Nacional de Investigadores) y del Programa para el Desarrollo Profesional Docente de Tipo Superior (PRODEP- Cuerpos Académicos) cuyo 
apoyo se visualiza a partir de financiamiento vinculado a la productividad de la comunidad científica. Un tercer referente será la asociación voluntaria al Consejo Mexicano de Investigación Educativa, instancia cuyo origen se sitúa en los años ochenta, casi en paralelo al Sistema Nacional de Investigadores (S.N.I).

¿Qué sentido tiene realizar un estudio exploratorio descriptivo de los investigadores educativos para una institución como la BUAP? En primer lugar, si bien se tienen indicadores que forman parte de los reportes de la universidad al gobierno federal 0 , en su defecto, se integran a los informes rectorales éstos son generales, entonces, resulta necesario considerar reportes sistemáticos de comunidades cuyos trabajos atienden características de un campo como es la educación, temática imprescindible a los propósitos de la universidad, y todavía más con las peculiaridades que guarda el nivel educativo superior con necesidades expresas y soluciones de largo aliento. A la fecha no existen reportes sistemáticos que nos digan si ha incrementado el número de miembros, ¿a qué unidades se adscriben?, ¿qué formación tienen? ¿cuáles son sus líneas de investigación?, ¿cuáles han sido sus problemáticas para constituirse? ¿Transitar del trabajo individual a uno colectivo qué ventajas o desventajas les representa? Lo que investigan se vincula con problemáticas propias de la educación superior y del ámbito donde se desempeñan. Tener respuestas a lo anterior sería provechoso para la BUAP debido a que podría considerarse como referente las investigaciones y propuestas de dicha comunidad.

Es posible que no todas las preguntas puedan ser respondidas de forma contundente en este primer ejercicio exploratorio lo que si se puede afirmar es que la comunidad de investigadores educativos de la BUAP ha incrementado su número considerablemente a partir de los apoyos derivados de programas federales cuyas exigencias de habilitación los mantiene en status diferenciados.

En párrafos anteriores hemos venido mencionando dos conceptos que son clave para el desarrollo del estudio exploratorio: investigación educativa e investigador educativo. En cuanto al primer concepto se alude a los trabajos realizados por la Red Mexicana de Investigadores sobre la Investigación Educativa (REDMIIE) quienes definen a la investigación educativa como una práctica científica intencionada, social e histórica que tiene como objeto de estudio la educación en su acepción amplia: incluye sujetos, procesos, relaciones, instituciones, situaciones y prácticas para responder a las preguntas qué, quién, cómo, cuándo, dónde, por qué y para qué, a través de la producción, difusión y distribución del conocimiento con la finalidad de comprender, explicar y mejorar la educación. Considerando que existen normas y reglas que las comunidades establecen para llevar a cabo dicha investigación, así como subrayar que también responde a distintas tradiciones epistémicas donde se requiere sistematicidad, creatividad e intuición heurística (López et al., 2013).

Para la Asociación Americana de Investigación Educativa (AERA siglas en inglés) de manera particular enfatiza que la academia vía la investigación busca describir, entender y explicar cómo el aprendizaje toma lugar en la vida de las personas y cómo los contextos educativos formales y no formales afectan las formas de aprendizaje y coincide con el COMIE en el sentido que la investigación en el ámbito educativo abarca el espectro completo de los métodos rigurosos y apropiados (AERA, 2015). McWilliam y Lee (2006) afirman que resulta un imperativo para los gobiernos establecer políticas en pro de la investigación educativa. Investigar en general y, por supuesto en educación, requiere una formación que considera los estudios de posgrado así como los apoyos en términos de financiamiento. Para McWilliam y Lee (2006) se debe tener presente que por sí mismos los hallazgos de la investigación educativa no resuelven los problemas que enfrentan las instituciones, debe conjuntarse con la voluntad de hacer uso de éstos para plantear soluciones concretas.

Definida la investigación educativa, cabe preguntarse a quiénes se considera investigadores educativos. Atendiendo a las aportaciones del COMIE, se define al investigador educativo como la persona que realiza actividades metódicas de indagación orientadas a la generación de nuevo conocimiento y al desarrollo del campo educativo. Cuenta con obra publicada y reconocimiento de sus pares en el campo de la investigación educativa o por programas institucionales de fomento a la investigación en cualquiera de sus modalidades o niveles (López et al., 2013). Siguiendo tal idea, se puede considerar que el COMIE ha llevado a cabo ese reconocimiento de pares, así como los programas de apoyo federal a la investigación (S.N.I y PRODEP) cuyos parámetros de referencia han incidido para transitar del trabajo individual al colaborativo entre los investigadores educativos.

El Consejo Mexicano de Investigación Educativa se configuró en 1993, doce años después del Primer Congreso de Investigación Educativa llevado a cabo en 1981. Es una institución que reúne a los investigadores profesionales del más alto nivel con el objetivo de promover la investigación educativa dentro de los estándares científicos de calidad. Ha contribuido de manera consistente y continua a la identificación de los problemas educativos del país así como de sus soluciones (COMIE, 2015). Actualmente cuenta con 467 investigadores pertenecientes tanto a universidades públicas como particulares, siendo originarios de 28 estados del país (COMIE, 2015). Ha de subrayarse que es la primera institución en el país en construir 
los estados del conocimiento en relación a las diferentes temáticas educativas y su evolución. Los estados del conocimiento de 1992 a 2002 y 2002 a 2012 elaborados por los mismos socios quienes se suscriben en redes de colaboración. Adherirse al COMIE no es obligatorio, sin embargo, pertenecer a esta asociación implica tener un posgrado en el área educativa y haber publicado en los últimos cinco años, libros, capítulos de libros y artículos tanto en revistas indizadas como arbitradas.

Durante la década de los años ochenta, con objeto de que las instituciones pudieran retener a más investigadores y profesores destacados, el gobierno federal, por recomendación de la Academia de la Investigación Científica A.C. creó el Sistema Nacional de Investigadores (OCDE, 1997). A partir de 1984, el Sistema Nacional de Investigadores reconoce la labor de las personas dedicadas a producir conocimiento científico y tecnología. El reconocimiento se otorga a través de la evaluación por pares y consiste en otorgar el nombramiento de investigador nacional. Esta distinción simboliza la calidad y el prestigio de las contribuciones científicas en paralelo al nombramiento se otorgan estímulos económicos cuyo monto varía con el nivel asignado (CONACYT, 2015). Los niveles considerados son cuatro: candidato, nivel I, II, y III cuyas condiciones de logro también atienden a la calidad y alcance de las publicaciones así como a la formación de recursos humanos. En cuanto a la categoría candidato se requiere tener el grado de doctor, demostrar capacidad para llevar a cabo investigación científica o tecnológica, además, no haber pasado más de quince años entre el término de la licenciatura y la obtención del grado de doctor. En relación al nivel I los requisitos incluyen tener el grado de doctor, haber realizado investigación científica o tecnológica creativa y original expresada en publicaciones o patentes, además de haber dirigido tesis de licenciatura 0 posgrado así como formar recursos humanos. Los investigadores nivel II habrán de cumplir los requisitos del nivel I y además de demostrar que sus investigaciones demuestran la consolidación de una línea de investigación. El nivel III exige cumplir con los requisitos del nivel II y además llevar a cabo investigación científica o tecnológica trascendente para generación y aplicación del conocimiento (CONACYT, 2012). A estas nuevas formas de ver el conocimiento, se agregó que ya no era posible sostener el mito del investigador solitario. Esto se expresó en nuevas formas de asociarse y co-construir el conocimiento situación en la que algunas disciplinas académicas se destacan más que otras por la tradición que mantienen de trabajar en grupo (Orland-Barak y Tillema, 2007).

El Programa para el Desarrollo Profesional Docente de Tipo Superior (PRODEP antes PROMEP) que en 1996 fue formulado con el propósito de contribuir para que los Profesores de Tiempo Completo (PTC) de las instituciones públicas de educación superior alcanzaran las capacidades para realizar investigacióndocencia, se profesionalizaran, se articularan y se consolidaran en cuerpos académicos. Cabe señalar que los apoyos del PRODEP pueden considerarse de dos tipos, aquéllos que apoyan la formación de los profesores de manera individual, (para que el lector tenga una idea en 1994 en el país sólo el 22\% de 67, 355 profesores tenían estudios superiores a los de licenciatura, nivel en el que se desempeñaban) (OCDE, 1997). Y aquéllos apoyos destinados a los cuerpos académicos, entendiéndose por estos últimos al conjunto de profesores-investigadores que comparten una o más líneas de estudio, cuyos objetivos y metas están destinados a la generación y/o aplicación de nuevos conocimientos. Pueden clasificarse como: a) consolidados, b) en consolidación y c) en formación (SEP, 2015a).

Dentro de las características de los consolidados se mencionan: La mayoría de sus integrantes tienen la máxima habilitación académica que los capacita para generar o aplicar innovadoramente el conocimiento de manera independiente. Cuentan con amplia experiencia en docencia y en formación de recursos humanos. La mayoría cuenta con el reconocimiento de perfil deseable, tienen un alto compromiso con la institución, colaboran entre sí y su producción es evidencia de ello. Demuestran una intensa actividad académica manifiesta en congresos, seminarios, mesas y talleres de trabajo, etc., de manera regular y frecuente, con una intensa vida colegiada, y sostienen una intensa participación en redes de intercambio académico con sus pares, en el país y en el extranjero, así como con organismos e instituciones nacionales y del extranjero (SEP, 2015b).

Los cuerpos académicos en consolidación tienen las siguientes características: Más de la mitad de sus integrantes tiene la máxima habilitación y cuentan con productos de generación o aplicación innovadora del conocimiento. La mayoría de ellos tiene reconocimiento del perfil deseable. Participan conjuntamente en líneas de generación o aplicación innovadora del conocimiento bien definidas. Por lo menos, la tercera parte de quienes lo integran cuenta con amplia experiencia en docencia y en formación de recursos humanos. Colaboran con otros Cuerpos Académicos (SEP, 2015b). En relación a los cuerpos académicos en formación: Tienen identificados a sus integrantes, de los cuales al menos la mitad tiene el reconocimiento del perfil deseable. Tienen definidas las líneas de generación o aplicación del conocimiento e identificados algunos Cuerpos Académicos afines, y de alto nivel, de otras instituciones del país o del extranjero con quienes desean establecer contactos (SEP, 2015b). Se considera al perfil deseable una condición de equilibrio entre las cuatro actividades que realiza un profesor investigador: docencia, tutoría, investigación y gestión. 
Como se puede apreciar la conformación de una comunidad de investigadores educativos inicia desde los años ochenta con las iniciativas del COMIE, aunque hubo de esperar una década más para consolidar dicho proyecto que a la fecha cuenta con 13 congresos nacionales mismos que reúnen cada dos años a un número considerable de especialistas. En la misma década de los ochenta, el S.N.I fortalecería la investigación al ser el primer programa que evaluaría las trayectorias individuales y así apoyar con incentivos económicos adicionales al salario base de los académicos, tomando en cuenta los logros que se tenían en investigación. Casi al final de los años noventa el PROMEP hoy PRODEP impulsaría el paso del trabajo individual al colaborativo sustentado en cuatro ejes: docencia, tutoría, investigación y gestión. Durante más de dos décadas quienes ya habían empezado un camino en la investigación con los apoyos del S.N.I., tuvieron que implicarse en un esfuerzo colaborativo bajo el PRODEP que no sería fácil poner en marcha y que tendría que irse ajustando a las circunstancias de cada universidad y a los intereses de cada investigador.

Previo al estudio exploratorio que llevamos a cabo, se identificaron las circunstancias documentadas de la BUAP de las primeras acciones en relación a llevar a cabo investigación educativa así como la conformación de una comunidad de investigadores educativos. A pesar de los difíciles embates económicos y políticos durante la década de los años ochenta en México y, aun cuando algunos centros desaparecieron debido a la falta de presupuesto, fue en 1983 cuando en la Universidad Autónoma de Puebla se concibió el Centro de Estudios Universitarios (CEU) órgano que se encargaría de analizar diversos aspectos de la educación superior y con ello tomar medidas encaminadas a mejorar el quehacer universitario, entre otros tópicos, estudiaría las formas internas de gobierno, la relación de las universidades con su entorno, los planes de estudio y las reformas curriculares. Fue en 1986 cuando el Centro iniciaría formalmente sus actividades (Garrido 2009). Como sugiere Martínez (1996) la educación superior vio mejores épocas en la década de los noventa en términos de financiamiento lo que llevó a otros logros a dicho Centro. Por ejemplo, la apertura de los estudios de maestría en Educación Superior. Después este Centro pasaría a la Facultad de Filosofía y Letras en 1998 junto con el posgrado que ofertaba.

En el período de 1993 a 2001, el COMIE llevó a cabo una investigación donde la Benemérita Universidad Autónoma de Puebla (BUAP) presentaba avances significativos si se atiende a los 24 investigadores que lograron entrevistar en distintas dependencias de la institución, asociado este número a la creación de algunos programas de posgrado vinculados a la educación. Siguiendo tal idea sólo 6 pertenecían al Sistema Nacional de Investigadores (Royo et al., 2003). De acuerdo a Sandoval et al., (2014) un esfuerzo a partir de la Red Mexicana de Investigadores de la Investigación Educativa (Redmiie) permitió configurar diagnósticos acerca de quiénes son los que investigan, qué investigan, y los usos que se han dado a estos resultados en 15 estados de la república mexicana. Si bien aparece el diagnóstico para el estado de Puebla, las instituciones comprometidas con este ejercicio fueron cuatro, todas de carácter privado. Lamentablemente la BUAP no se integró en dicho diagnóstico, lo que deja al estado con datos cortos al ser la universidad pública y encontrarse ausente.

\section{METODOLOGÍA}

El estudio es de tipo exploratorio descriptivo. Cabe destacar que preferimos seguir el camino de la revisión de las bases de datos de tres referentes nacionales porque ellos han incidido en la consolidación de la investigación educativa en distintas formas, además resulta complejo para los agentes investigadores obtener apoyos que no provengan sobre todo de los recursos federales como S.N.I y PRODEP.

La exploración no respondió a la lógica de creación en tiempo de las instancias a que se ha hecho referencia. Se recordará que primero fue el trabajo individual que caracterizó al ámbito de la investigación educativa, sin embargo, decidimos comenzar la revisión por las bases de datos de PRODEP (cuerpos académicos) debido a que permite la inclusión de profesores que apenas incursionan en la investigación educativa. De ahí, se contrastó cuántos y quiénes de los 116 pertenecen al S.N.I. Las bases fueron monitoreadas durante 8 meses (julio, 2014-febrero 2015). Durante el monitoreo las bases fueron modificadas algunas ocasiones por los procesos de evaluación, nuevas adhesiones o bajas que ocurren durante algunos períodos en las instituciones. En el caso del COMIE, de igual manera, se filtró la base, así pudimos saber cuántos pertenecen al estado de Puebla, cuántos forman parte de la BUAP y están incorporados también a las otras instancias.

Revisar las tres bases de datos nos permitió tener claridad en los siguientes aspectos: PRODEP sólo incluye a los investigadores que son tiempos completos así que de haber académicos abocados a investigar en educación cuyas categorías laborales sea por horas o medio tiempo no son considerados ya que no podrían por ello ser parte del núcleo básico de un cuerpo académico debido a que se requiere ser tiempo completo según las reglas de PRODEP. Sin embargo, la categoría laboral no es un impedimento para el ingreso al S.N.I o el registro al COMIE. Hemos sido exhaustivos en las revisiones pero no ignoramos este tipo de inconvenientes. 


\section{RESULTADOS Y DISCUSIÓN}

Al revisar la base de datos de PRODEP identificamos que la BUAP tiene registrados un total de 189 cuerpos académicos, de los cuales 26 están asociados a la investigación educativa bien sea en su primera, segunda o tercera línea de trabajo lo que representa el 14\% del total (ver tabla 1). Forman parte de estos 26 cuerpos académicos un total de 116 profesores de tiempo completo.

La BUAP actualmente está conformada por 30 unidades académicas, integradas de la siguiente forma: 2 escuelas, 23 facultades y cinco institutos (en éstos últimos la actividad primordial es la investigación aunque también hay programas de posgrado que se ofertan). Cabe destacar que existen investigadores educativos adscritos a trece unidades académicas, esto es más allá de ser ubicados en el área de Educación, Humanidades y Arte, por ejemplo, vale la pena señalar los grupos identificados en las Facultades de Ingeniería, Ciencias de la Computación y Físico-Matemáticos, por cierto, en esta última facultad el cuerpo académico es reconocido por su producción alrededor de los problemas y propuestas en cuanto a la enseñanza y aprendizaje de las matemáticas.

Si se atiende a la explicación sobre lo que significa consolidación es el grupo que caracteriza el mayor nivel de experiencia, es decir, su producción académica se ve representada por libros, capítulos de libros, artículos en revistas nacionales e internacionales bajo arbitraje, además de formar recursos humanos a nivel de posgrado principalmente. Sin faltar la vinculación en redes. Dentro de este grupo de 47 investigadores 21 son parte del Sistema Nacional de Investigadores lo que representa un 45\%. Cabe hacer notar que en este grupo de consolidados se ubica un investigador cuya producción lo acredita como el referente mexicano más leído en lengua inglesa nivel III en el S.N.I. El $90 \%$ de los investigadores que son parte del S.N.I se encuentra en el nivel I. De igual manera se puede decir que el cuerpo denominado Etnocoreología y Etnomusicología es el único que no tiene integrantes en el S.N.I., sin embargo, su nivel de consolidación señala la habilitación necesaria y el fuerte trabajo en grupo que se demuestra con producción y acciones académicas colectivas. En este grupo de cuerpos consolidados, se identificaron tres investigadores que pertenecen al COMIE.

En el segundo grupo que se denomina cuerpos en consolidación se identificaron 51 agentes activos, de los cuales 13 pertenecen al S.N.I, es decir, el 25\%. Como su nombre lo enuncia, estos investigadores tienen ya un camino andado en el sentido de la producción de artículos, capítulos de libros y libros, sin embargo, sólo una tercera parte o menos está habilitada para la formación de recursos humanos. Por cierto, sólo tres se identifican como parte del COMIE (ver tabla 1).

En el tercer grupo, los cuerpos académicos en formación registran a 18 investigadores y sólo uno en el S.N.I. Son considerados noveles en el sentido que deben trabajar todavía más para su habilitación como investigadores. Quizá de manera individual su producción académica les permite tener el reconocimiento de Perfil Deseable, sin embargo, su producción colectiva no es la suficiente. Ninguno en dicho grupo está afiliado al COMIE (ver tabla 1).

Las líneas de generación y aplicación del conocimiento nos indican fuertes intereses en varias áreas que resultan primordiales en la educación superior: desde distintas disciplinas se investigan los problemas asociados con la enseñanza y el aprendizaje, así como la formación profesional del universitario y la pertinencia que ésta pueda tener. De las líneas también se infiere el interés por estudiar las políticas educativas, quizá, en su momento, vinculadas a la organización y desarrollo institucional. La formación docente es punto común para varios cuerpos académicos, sobre todo, en el área de lenguas. Sin faltar por supuesto algunas líneas vinculadas al desarrollo de la tecnología y los entornos virtuales de aprendizaje.

Después de haber revisado tres bases de datos diferentes se puede inferir que las dos primeras atienden en sus categorías de manera directa a procesos evaluativos que permiten ubicar a los cuerpos académicos e investigadores en alguna de éstas. Esto es son programas derivados de políticas públicas que jerarquizan el desempeño de los investigadores. Cabe señalar que acceder a este tipo de programas no sólo depende del nivel de producción de los investigadores sino del tipo de contratación que mantienen. Por ejemplo, puede darse el caso de académicos que no son tiempos completos, pero que formen parte del S.N.I y del COMIE. Sin embargo, no podrán formar parte del núcleo básico de un cuerpo académico. La revisión y análisis de quién investiga, dónde se encuentra y qué temáticas aborda, considerando tres bases de datos diferentes, nos señala que la investigación educativa va más allá de pensarse en Educación y Humanidades, existen 13 unidades académicas donde los profesores investigadores con otras formaciones, por ejemplo, doctores en física, ingeniería, historia, sociología, etc. llevan a cabo labores de investigación en este campo. 
Tabla 1. Agentes investigadores (PRODEP, S.N.I. y COMIE).

\begin{tabular}{|c|c|c|c|c|c|c|}
\hline Unidad de Adscripción & $\begin{array}{l}\text { (PRODEP) Cuerpos } \\
\text { Académicos Consolidados }\end{array}$ & $\begin{array}{c}\text { No. } \\
\text { Integrante } \\
\mathrm{S}\end{array}$ & Hombres & Mujeres & S.N.I. & COMIE \\
\hline $\begin{array}{l}\text { Instituto de Ciencias Sociales y } \\
\text { Humanidades }\end{array}$ & Historia de la cultura & 3 & 1 & 2 & 3 & 0 \\
\hline Facultad de Físico Matemáticas & $\begin{array}{l}\text { Aprendizaje y enseñanza de } \\
\text { las ciencias }\end{array}$ & 9 & 4 & 5 & 4 & 0 \\
\hline Facultad de Lenguas & $\begin{array}{l}\text { Investigación, literacidad y } \\
\text { desarrollo docente }\end{array}$ & 4 & 0 & 4 & 1 & 0 \\
\hline Escuela de Artes & $\begin{array}{l}\text { Etnocoreología y } \\
\text { Etnomusicología }\end{array}$ & 6 & 5 & 1 & 0 & 0 \\
\hline $\begin{array}{l}\text { Facultad de Cs. de la } \\
\text { Computación }\end{array}$ & $\begin{array}{l}\text { Entornos colaborativos } \\
\text { digitales }\end{array}$ & 7 & 1 & 6 & 3 & 1 \\
\hline Facultad de Filosofía y Letras & Estudios Históricos & 5 & 2 & 3 & 3 & 0 \\
\hline Facultad de Administración & $\begin{array}{l}\text { Investigación científica, } \\
\text { desarrollo tecnológico y } \\
\text { vinculación social }\end{array}$ & 4 & 1 & 3 & 2 & 2 \\
\hline $\begin{array}{l}\text { Facultad de Cs .de la } \\
\text { Computación }\end{array}$ & $\begin{array}{l}\text { Algoritmos Combinatorios y } \\
\text { Aprendizaje }\end{array}$ & 6 & 4 & 2 & 2 & 0 \\
\hline Facultad de Economía & $\begin{array}{l}\text { Trabajo y conocimiento en el } \\
\text { desarrollo latinoamericano }\end{array}$ & 3 & 2 & 1 & 3 & 0 \\
\hline Subtotales & & 47 & 20 & 27 & 21 & 3 \\
\hline & $\begin{array}{c}\text { Cuerpos académicos en } \\
\text { consolidación }\end{array}$ & & & & & \\
\hline Facultad de Lenguas & $\begin{array}{l}\text { Docencia e innovación } \\
\text { profesional }\end{array}$ & 5 & 2 & 3 & 1 & 0 \\
\hline Facultad de Medicina & Sociomedicina & 5 & 3 & 2 & 1 & 0 \\
\hline Facultad de Contaduría Pública & Desarrollo Institucional & 5 & 0 & 5 & 0 & 0 \\
\hline Facultad de Filosofía y Letras & Lingüística & 3 & 2 & 1 & 0 & 0 \\
\hline Instituto de Ciencias & Desarrollo Sustentable & 4 & 3 & 1 & 0 & 0 \\
\hline $\begin{array}{l}\text { Instituto de Ciencias Sociales y } \\
\text { Humanidades }\end{array}$ & $\begin{array}{l}\text { Desarrollo de habilidades } \\
\text { cognitivas y discursivas }\end{array}$ & 4 & 1 & 3 & 2 & 1 \\
\hline $\begin{array}{l}\text { Facultad de } \\
\text { Contaduría Pública }\end{array}$ & $\begin{array}{l}\text { Educación, Empresa y } \\
\text { Humanidades }\end{array}$ & 3 & 0 & 3 & 0 & 0 \\
\hline Escuela de Artes & Música & 3 & 2 & 1 & 0 & 0 \\
\hline Facultad de Ingeniería & $\begin{array}{l}\text { Innovación educativa en } \\
\text { ingeniería }\end{array}$ & 3 & 3 & 0 & 0 & 0 \\
\hline Facultad Filosofía y Letras & $\begin{array}{l}\text { Estructura, formación y } \\
\text { práctica profesional }\end{array}$ & 3 & 2 & 1 & 2 & 1 \\
\hline Facultad de Físico Matemáticas & Análisis Matemático & 4 & 4 & 0 & 4 & 0 \\
\hline Facultad de Filosofía y Letras & Educación Superior & 4 & 1 & 3 & 1 & 1 \\
\hline Instituto de Ciencias & $\begin{array}{l}\text { Educación en Ciencias e } \\
\text { investigación en nuevos } \\
\text { materiales }\end{array}$ & 5 & 3 & 2 & 2 & 0 \\
\hline Subtotales & & 51 & 26 & 25 & 13 & 3 \\
\hline & $\begin{array}{l}\text { Cuerpos Académicos en } \\
\text { formación }\end{array}$ & & & & & \\
\hline Facultad de Arquitectura & $\begin{array}{l}\text { Diseño Estratégico e } \\
\text { Innovación para la } \\
\text { comunicación visual }\end{array}$ & 3 & 0 & 3 & 0 & 0 \\
\hline Facultad de Arquitectura & $\begin{array}{l}\text { Didáctica del Urbanismo y } \\
\text { Diseño Ambiental }\end{array}$ & 6 & 4 & 2 & 0 & 0 \\
\hline Facultad de Lenguas & Estudios críticos & 3 & 1 & 2 & 1 & 0 \\
\hline Facultad de Lenguas & Interacciones en el aula & 6 & 3 & 3 & 0 & 0 \\
\hline Subtotales & & 18 & 8 & 10 & 1 & 0 \\
\hline Total & & 116 & 54 & 62 & 35 & 6 \\
\hline
\end{tabular}


Tanto la Facultad de Lenguas, como la Facultad de Filosofía y Letras concentran a un número mayor de investigadores que el resto de las unidades. En el caso de PRODEP, la mayor parte de los cuerpos académicos se vinculan al exterior pero muy poco con sus pares internos. Aun cuando algunos convergen en las líneas de investigación que cultivan, por ejemplo, formación de profesores o estrategias de aprendizaje los vínculos en ese sentido también son escasos. Quizá porque se han llevado al extremo las reglas de operación del programa gubernamental, en el sentido que tiene un valor superior vincularse con el ámbito nacional o exterior, así parece. Sin embargo, como afirma Lin et al., (2015) para el caso de los Estados Unidos, en cuanto a la investigación educativa poco se ha indagado sobre tres aspectos que son medulares para valorar si un trabajo en redes vale el esfuerzo: a) la composición de la red, actores de la organización y los recursos con los que cuentan, b) estructura de la red y las conexiones entre actores y c) proceso y estrategias empleados para gobernar la acción colectiva de la red.

Difícilmente se encuentra un grupo conformado por investigadores de distintas unidades académicas, esto es todos los miembros son de la misma escuela, facultad o instituto. No permitiendo dar paso a una interdisciplinariedad más real y no sólo en el discurso. Una de los obstáculos que parecería no permite el trabajo interdisciplinario desde la conformación de los cuerpos académicos se debe a que el grupo una vez conformado debe estar suscrito a una unidad académica, misma que tendrá todos los beneficios que el grupo obtenga, así si se integra algún elemento de otra unidad académica no reportará beneficios directos para su escuela, facultad o instituto en términos de indicadores.

Aun cuando los cuerpos académicos en formación sólo son cuatro valdría preguntarse de qué manera estos grupos podrán desarrollarse y pasar de su condición de noveles a lograr otros niveles de experiencia. Quizá también es posible pensar que los gobiernos asignan los apoyos pretendiendo que los resultados de investigación se obtengan de manera cada vez más rápida, al no conseguirlo estos grupos se ven menos incentivados, por lo tanto, los apoyos son menores. Parker advierte que los gobiernos centrales cada vez más están declinando los apoyos y solo concederlos ante las expresiones de eficiencia y eficacia, por lo menos existe evidencia desde Europa hasta Asia, incluso se presiona para obtener recursos de otras fuentes (Araneda et al., 2013).

La comunidad de 116 investigadores educativos aun con status diferenciados ha reafirmado en crecimiento lo que ya en 2003 se subrayaba, el número de investigadores educativos se había duplicado en relación a la década anterior principalmente en las entidades federativas fuera de la zona metropolitana (López et al., 2013). En esta segunda década ese crecimiento es mayor. La información obtenida servirá de base para generar un directorio que permita incluir a todos los agentes activos, no importando su status, al mismo tiempo se puede inferir bajo qué apoyos de política generan ese conocimiento. Cabe señalar que queda sólo bajo el interés de los propios investigadores educativos reflexionar acerca de la constitución del campo y sus agentes. Las bases de datos están abiertas sólo falta precisar categorías y ser persistente para buscar la información. Bases que sirven de ejes para una universidad de las dimensiones de la BUAP. Esperamos que en el siguiente estado del conocimiento de la investigación de la investigación educativa 2012-2022 del COMIE los investigadores educativos de la BUAP tengamos información disponible y organizada que reportar. Incluso ya considerar qué tipo de producción se realiza, cuáles son las metodologías más recurrentes, cómo se difunden los resultados y cuáles son los impactos. Ayudaría incluso para saber qué otras posibilidades de vínculos pueden promoverse dentro de la misma institución. Especialmente cuando las formas de comunicación actualmente lo permiten.

\section{CONCLUSIONES}

Trece años han pasado desde que apareció el documento "El campo de la investigación educativa en México: usos y coordinación" (2003) y donde se hizo referencia a los 24 investigadores que en esa época conformaban la comunidad de investigadores educativos de la BUAP, además que sólo 6 pertenecían al S.N.I. y 3 al COMIE. Actualmente la comunidad tiene otros alcances que son resultado de varios factores. Entre ellos, las directrices que han marcado las políticas de gobierno desde la década de los años ochenta hasta la fecha, y que si bien han implicado apoyos para la formación y habilitación de investigadores, también se ha considerado el tránsito del trabajo individual al trabajo colaborativo, ejercicio que ha tomado su tiempo para mostrar logros. La presencia de 116 académicos abocados a realizar investigación educativa nos muestra que en la universidad existe un interés genuino en indagar sobre temáticas vinculadas a los procesos de enseñanza-aprendizaje así como en la formación de profesores, organización y desarrollo institucional no dejando de lado el uso de la tecnología y los entornos colaborativos virtuales. Esto confirma lo anteriormente expresado, la investigación se contextualiza llevando en ello la institución ciertas ventajas. Organizados en cuerpos académicos se advierte que no sólo participan las facultades que cuentan con algún posgrado en educación sino de 13 unidades académicas que exploran sobre sus propias experiencias. Incluso existen expresiones únicas en el país como lo es el cuerpo académico de Etnocoreología y Etnomusicología dedicado a estudiar las expresiones dancísticas de carácter identitario que realizan las culturas populares y los grupos indígenas de México con el ánimo de formar transductores dancísticos. 
No menos importante resulta que la mayor parte de los cuerpos académicos han permanecido en actividades conjuntas a lo largo de varios años y han incrementado su nivel de consolidación, tarea que no resulta sencilla pues el trabajo colaborativo que implica exige responsabilidades y beneficios compartidos. Por ejemplo cuando este estudio exploratorio se llevó a cabo los cuerpos académicos de Sociomedicina, Desarrollo Sustentable y Educación Empresa y Humanidades se mantenían como cuerpos académicos "en consolidación", significaba que, sólo la mitad de sus miembros, contaban con la habilitación necesaria, es decir, tener los grados como doctores y además publicar de manera colegiada y además participar en la formación de recursos humanos. Estos tres grupos han modificado su status y a la fecha ya están en la categoría de consolidados. La transformación puede significar en tiempo una década de trabajo.

De los 116 investigadores 35 son parte del S.N.I porcentaje que se incrementa con lentitud debido a que las acciones en colaboración parecen no coadyuvar en cuanto a los requerimientos solicitados a nivel individual. Se advierte que de 35, 21 investigadores del S.N.I se ubican en los cuerpos académicos consolidados donde parecen converger los parámetros de exigencia de ambas instancias.

No sorprende el número de investigadores educativos con registro en el COMIE, sólo nueve de los cuales dos no forman parte de cuerpo académico alguno y un tercero que recién participa en un cuerpo en consolidación. Baste recorder que la afiliación es voluntaria, a diferencia de las otras instancias. Sin embargo, la asistencia a los congresos organizados y la inserción de artículos a la Revista Mexicana de Investigación Educativa editada por el COMIE es un hecho.

La comunidad de 116 investigadores educativos en la BUAP es una realidad que se ve definida no sólo por los intereses de investigación sino también por las propuestas que realizan y que la institución puede aprovechar. Dicha comunidad también se ha constituido a partir de criterios externos lo que la mantiene bajo status diferenciados tanto de manera individual así como colectiva, condiciones que nos permiten reconocer sus habilitaciones y alcances académicos.

\section{REFERENCIAS}

Aera. What is education research? http://www.aera.net/EducationResearch/WhatisEducationResearch/ Acceso 12 Mayo (2015)

Altbach, P. Peripheries and Centres: Research Universities in Developing Countries, Higher Education and Management and Policy: 19 (2), 111-134 (2007)

Araneda, C., Rodríguez, E., Pedraja, L. Relación entre el financiamiento fiscal, la calidad del cuerpo académico y la retención de estudiantes universitarios en Chile, doi: 10.4067/S0718-50062013000600006, Form. Univ. vol.6 (6), pp.55-64 (2013)

CENEVAL. Perfil Institucional e Investigaciones, http://www.ceneval.edu.mx/ceneval web/content.do. Acceso 10 de Febrero (2016)

COMIE. La investigación educativa en México: usos y coordinación, revista@comie.org.mx; ISSN: 14056666, Revista Mexicana de Investigación Educativa, 8(19), 847-898 (2003)

COMIE. Acerca del Comie, http://comie.org.mx/v4/secciones/historia. Acceso 5 Mayo (2015)

CONACYT. Sistema Nacional de Investigadores. ¿Qué es? http://conacyt.gob.mx/index.php/elconacyt/sistema-nacional-de-investigadores. Acceso 18 de Febrero (2016)

CONACYT. Reglamento del S.N.I. Diario Oficial. México, D.F. (2012)

Garrido, C. Vicerrectoría de Investigación y Estudios de Posgrado. Tiempo Universitario. Gaceta histórica de la BUAP. Año 12, número 10. BUAP, Puebla, México (2009)

INNE. ¿Qué es el INEE?, http://www.inee.edu.mx/index.php/acerca-del-inee/que-es-el-inee. Acceso 18 de Febrero (2016).

Lin, J., Meredith, J., Chids, J., Stein, M., Weber, D. Designing Intern-Organizational Networks to Implement Education Reform: An Analysis of State Race to the Top Applications, doi: 10.3102/0162373714527341, Educational Evaluation and Policy Analysis, (en línea). 37(1), 92-112 (2015)

López, M., Sañudo, L., Maggi, R. Introducción, en Investigaciones sobre la Investigación Educativa 20022011,1ª edición. 21-55, ANUIES - COMIE, México, D.F. México (2013) 
Martínez, F., La investigación educativa en México en el contexto latinoamericano, La investigación educativa en el mundo. Con un capítulo especial sobre el caso México, pp. 347-372 Fondo de Cultura Económica, Distrito Federal, México (1996)

McWilliam, E., Lee, A. The problem of the problem with the educational research, The Australian Educational Researcher: 33(2), 43-60 (2006).

OCDE. Exámenes de las Políticas Nacionales de Educación. 1ª̣. Edición, 3-245. Organización para la Cooperación y el Desarrollo Económicos. Distrito Federal, México (1997)

Orland-Barak, L., Tillema, H. Researchers' Construction of Knowledge from Studying Professional Conversation Groups, The Educational Forum: 71 (Summer), 361-371 (2007)

Royo, I., Porras, L., Castillo, A. La investigación educativa en las universidades de Puebla, El campo de la investigación educativa, 1993-2001, pp. 513-560. Consejo Mexicano de Investigación Educativa. Distrito Federal, México (2003)

Sandoval, D., López, Y., Martínez, R. Diagnósticos de la investigación educativa, en Investigación sobre la investigación educativa. pp. 139-216, 2002-2011, ANUIES - COMIE, México, D.F., México (2013)

SEP. Acerca del PRODEP. Programa para el Desarrollo Profesional Docente de Tipo Superior, en http://dsa.sep.gob.mx/prodep.html. Acceso 6 Mayo (2015a)

SEP. Otros- Preguntas más frecuentes. Programa para el Desarrollo Profesional Docente de Tipo Superior, http://dsa.sep.gob.mx/cuerposacademicos.html. Acceso 11 de Mayo (2015b) 